\title{
Nuevas herramientas en análisis de supervivencia: Chironomus riparius frente a triclosán.
}

\section{New survival analysis tools: Chironomus riparius exposed to triclosan.}

Josune Urien ${ }^{1 *}$

\section{Resumen}

Chironomus riparius (Meigen, 1803) es un insecto díptero, y un bioindicador utilizado con frecuencia en ensayos de toxicidad acuática. El análisis de supervivencia constituye uno de los objetivos principales de estos ensayos, y es habitual recurrir a pruebas estadísticas sencillas como el conocido probit, integrado en los paquetes de software estadísticos más utilizados. Sin embargo, en los últimos años se ha empezado a extender la aplicación de modelos matemáticos más complejos que permiten recabar información más allá de las concentraciones letales. En el estudio descrito en este artículo se analizó la supervivencia de C. riparius frente a triclosán (TCS) mediante el modelo de umbral de tolerancia GUTS (General Unified Threshold Model of Survival), analizando sus ventajas y la posibilidad de ser utilizado en simulación de nuevos escenarios.

Palabras clave: Chironomus riparius, TCS, bioindicador, análisis de supervivencia, GUTS.

\section{Abstract}

Chironomus riparius (Meigen, 1803) is a Dipteran insect and a bioindicator often used in aquatic toxicity trials. One of the main aims of these trials is to carry out survival analyses and it is common practice to resort to simple statistical tests such as the well-known probit, integrated within the most widely used statistical software packages. Nevertheless, over recent years, the application of more complex mathematical models has become more

1 Departamento de Matemática Aplicada, UPV/EHU.

Centro Asociado UNED-Bergara.

*Correspondencia: mirenjosune.urien@ehu.eus 
widespread. These allow further data to be obtained, in addition to lethal concentrations. The study described in this paper analysed the survival of $C$. riparius exposed to triclosan (TCS) using the GUTS tolerance threshold model (General Unified Threshold Model of Survival), and evaluated the advantages of this model and the possibility of using it in the simulation of new scenarios.

Key words: Chironomus riparius, TCS, survival analysis, GUTS theory.

\section{Laburpena}

Chironomus riparius (Meigen, 1803) intsektu dipteroa da, eta adierazle biologiko gisa sarritan erabilia uren toxikotasun probetan. Biziraupen-analisia izaten da proba horien helburu nagusienetakoa, eta ohiko software estatistikoetan aurkitzen den probit izeneko teknika ezaguna erabiltzen da sarritan. Hala ere, azken urteetan eredu matematiko konplexuagoak erabiltzeko joera hedatu da, horien bidez kontzentrazio hilgarriez gain bestelako informazioak lortzen baitira. Artikulu honetan deskribatzen den ikerketan C. riparius-en triklosanarekiko biziraupena aztertzen da, GUTS (General Unified Threshold Model of Survival) toletarantzia-ataria aplikatuta, horren abantailak aztertu eta eszenatoki berriak simulatzeko aukeren aldetik.

Gako hitzak: Chironomus riparius, adierazle biologikoa, TCS, biziraupen-analisia, GUTS.

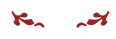

\section{Introducción}

En los últimos tiempos el aumento de las sustancias vertidas a los medios acuáticos ha hecho necesario un mayor control de la calidad de las aguas. Además de los habituales análisis físico-químicos se efectúa, entre otras medidas, un examen del estado de organismos seleccionados como indicadores biológicos o bioindicadores. Entre estos bioindicadores se encuentra Chironomus riparius (Meigen, 1803), un insecto díptero cuya fase larvaria transcurre en los sedimentos de los ríos.

C. riparius presenta varias particularidades que lo convierten en organismo de referencia en los ensayos de toxicidad: su amplia distribución, ser un elemento clave en la cadena trófica de los ecosistemas acuáticos (Berg \& Hellenthal, 1992), la facilidad de cultivo en laboratorio, su alta tolerancia a la contaminación orgánica y el déficit de oxígeno, o la presencia de hemoglobina en su estadio larvario, cuya concentración se modula ante la presencia de diferentes estresores mediambientales, lo que ha suscitado un creciente interés en su posible utilidad como biomarcador en monitoreo medioambiental (Moller Pilot, 2009; Nath, 2018).

Los ensayos de toxicidad con C. riparius suelen tener objetivos diversos, como el estu- 
dio de malformaciones y deformidades (Bhattacharyay et al., 2005; Planelló et al., 2015), o investigación a nivel molecular, bioquímico y celular (Park \& Choi, 2009; Morales et al., 2011; Wiseman et al., 2013). Las especificaciones de estos tests, consensuadas internacionalmente, se recogen en las guías publicadas por la OECD (OECD, 2004, 2010, 2011). El análisis de supervivencia es también un objetivo habitual, ya sea como paso previo al estudio de interés para elegir las concentraciones más adecuadas, o como objetivo en sí mismo. Con frecuencia se recurre a software estadístico especializado para realizar análisis de supervivencia conocidos, como el probit, que basándose en una distribución normal proporciona las concentraciones letales a tiempo fijado o LCx (por ejemplo, LC50 a 24 h sería la concentración de compuesto necesaria para que mueran la mitad de individuos en 24 h). No obstante, hoy en día la utilización de modelos matemáticos en diversas áreas científicas está llegando también a la ecotoxicología, proporcionando información que va mucho más allá de la mera obtención de las LCX.

El triclosán o TCS (5-cloro-2-(2,4-diclorofenoxi) fenol) es un compuesto aromático clorado utilizado como antimicrobiano tanto en el ambiente sanitario como doméstico (jabones, pasta dentífrica, cremas, etc.). Pasa a los sistemas acuáticos a través de las aguas residuales urbanas, ya que las plantas de tratamiento no lo eliminan en su totalidad (Dann \& Hontella, 2011). En seres humanos se ha detectado en orina, leche materna y sangre (Sandborgh-Englund et al., 2006.; Toms et al., 2011) y se ha descrito en varios estudios su potencial como disruptor endocrino, esto es, su capacidad de alterar el sistema endocrino (Chen et al., 2007; Gee et al., 2008). En cuanto a animales, se han reportado daños hepáticos y renales en roedores (Rodricks et al., 2010), alteraciones del sistema tiroideo en anfibios (Helbing et al., 2010), efectos teratogénicos en peces (Oliveira et al., 2009) y genotoxicidad en varias especies de invertebrados (Xu et al., 2015; Martínez-Paz et al., 2017).

La función de supervivencia $S(t)$, refleja la fracción de individuos supervivientes en función del tiempo. Toma por tanto valores comprendidos entre 0 y 1, ambos inclusive. Está relacionada con el denominado riesgo o hazard, $\mathrm{h}(\mathrm{t})$, que representa la probabilidad de morir (por unidad de tiempo) para un individuo que ha sobrevivido hasta el instante t:

$$
S(t)=\exp \left(-\int_{0}^{t} h(u) d u\right) \quad(E c .1)
$$

El cálculo de ambas funciones a través de modelos biológicos depende de los supuestos establecidos por cada modelo. La teoría GUTS (General Unified Threshold Model of Survival) o Modelo General Unificado de Umbral de Supervivencia, (Jager et al., 2011) se inicia con un modelo unicompartimental (Fig. 1, Ec.2) e incorpora los conceptos de Muerte Estocástica o SD (Stochastic Death) y Tolerancia Individual o IT (Individual Tolerance). 

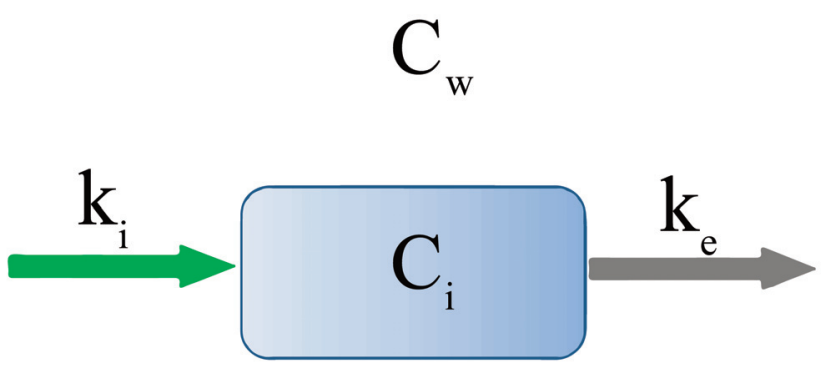

Fig. 1.- Modelo unicompartimental: $C_{w}$ es la concentración de xenobiótico en el medio, que penetra en el organismo con una tasa de absorción $k_{i}$ y lo abandona con una tasa de eliminación $k_{e}$, $C_{i}$ es la concentración interna.

Fig. 1.- One-compartment model: $C_{i}$ is the internal concentration, $C_{w}$ is the concentration in the exposure medium, $\mathrm{k}_{\mathrm{i}}$ is the absorption rate constant and $\mathrm{k}_{\mathrm{e}}$ is the elimination rate constant.

$$
\frac{d C_{i}(t)}{d t}=k_{i} C_{w}(t)-k_{e} C_{i}(t)
$$

Según el modelo SD, todos los individuos (larvas de C. riparius en nuestro caso) tienen la misma tolerancia a un compuesto dado. Para cada sustancia existe una concentración de no efecto, común para toda la población, a partir de la cual se incrementa la probabilidad de morir. Por lo tanto, el hecho de que a la misma concentración algunas larvas mueran y otras sobrevivan se debe simplemente a que las últimas han sido "más afortunadas". Por el contrario, el modelo IT supone que cada individuo posee su propia tolerancia individual, una concentración interna máxima a partir de la cual muere irremisiblemente (Fig. 2). Esta concentración máxima individual se distribuye en la población a través de una determinada función de densidad de probabilidad, que puede ser normal (campana de Gauss), log-logística, de Weibull, etc.

Para ambos modelos, IT y SD, la función de supervivencia se calcula partiendo de una función de riesgo individual:

$$
h_{z}(t)=k_{k} \max \left(0, C_{i}^{*}(t)-z\right)+h_{b}(t)
$$

Donde:

- $k_{k}$ es la tasa de muerte o killing rate y constituye la relación entre la concentración interna y el riesgo a morir por unidad de tiempo debido a la concentración interna de compuesto. 

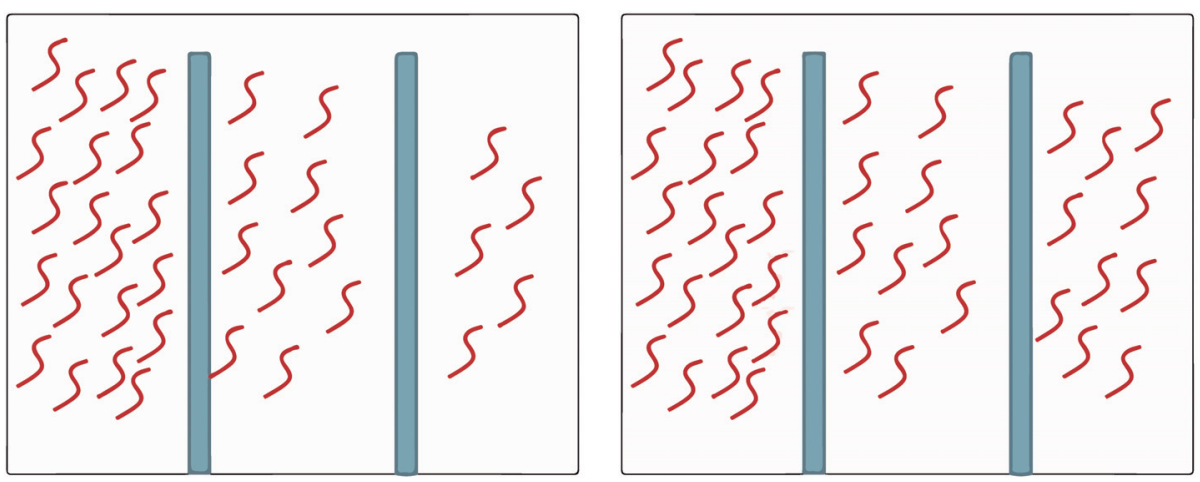

Fig. 2.- Izqda. el modelo SD: cada pulso (representado como barra azul) elimina la mitad de individuos cada vez. Dcha. el modelo IT: el primer pulso elimina los individuos más sensibles, un segundo pulso no produciría más muertes.

Fig. 2.- Left: SD model, each pulse (blue bars) eliminates half of the individuals. Right: IT model, first pulse kills the most sensitive individuals; following pulses will not cause more deaths.

- $h_{b}(t)$ es el riesgo basal, sin compuesto, representa la función de riesgo del control.

- z es el umbral de tolerancia, la máxima concentración de xenobiótico sin efecto en la supervivencia.

- $C_{i}^{*}(t)$ es la concentración interna escalada. Esta concentración se relaciona con la concentración interna a través del factor de bioconcentración mediante la expresión $C_{i}^{*}(t)=\frac{k e}{k i} C_{i}(t)$ y es sumamente útil cuando no se dispone de medios para medir la concentracion interna real, ya que reduce el número de parámetros a estimar (Jager et al., 2011).

El uso de la concentración interna escalada no persigue estimar el factor de bioconcentración, ni la constante de eliminación ke. De hecho esta constante ke, aun manteniendo su notación, habitualmente es denominada constante de tasa dominante, pues representa el proceso compensatorio dominante, el más lento, ya sea la propia eliminación o el proceso de recuperación del daño ocasionado por el compuesto (Jager et al., 2011, Kulkarni et al., 2013).

A partir de este riesgo individual se obtiene la función de supervivencia de un individuo con tolerancia z:

$$
S_{z}(t)=\exp \left(-\int_{0}^{t} h_{z}(T) d T\right)
$$


Introduciendo la densidad de probabilidad de dicha tolerancia, $f(z)$, se halla la función de supervivencia de toda la población:

$$
S(t)=\int_{0}^{\infty} S_{z}(t) f(z) d z
$$

En el supuesto de IT esta expresión se convierte en (Jager et al, 2011):

$$
S(t)=\int_{\max _{0<T<t} C_{i}^{*}(T)}^{\infty} f(z) d z=\left(1-F\left(\max _{0<T<t} C_{i}^{*}(T)\right)\right)
$$

Donde $F\left(\max _{0<T<t} C_{i}^{*}(T)\right)$ es la función de distribución de probabilidad elegida, aplicada en el máximo de concentración interna escalada. En los ensayos de laboratorio la concentración $\mathrm{k}_{\mathrm{e}}$

$$
C_{i}^{*}(t)=C_{w}\left(1-\exp \left(-k_{e} \cdot t\right)\right)
$$

y la expresión de la supervivencia se simplifica:

$$
S(t)=\int_{C_{i}^{*}(T)}^{\infty} f(z) d z=\left(1-F\left(C_{i}^{*}(T)\right)\right)
$$

Si se tiene en cuenta el riesgo basal:

$$
S(t)=\left(1-F\left(C_{i}^{*}(T)\right)\right) \cdot e^{-h_{b} t}
$$

Para el supuesto de SD la expresión de la función de supervivencia se convierte en (Jager et al, 2011):

$$
S(t)=\exp \left(-\int_{0}^{t} h_{z}(T) d T\right)
$$

Tras realizar la integración correspondiente se llega a:

$$
S(t)=\left\{\begin{array}{lr}
\exp \left(-h_{b} t\right) & \text { mientras } C_{i}^{*}(t)<z \\
\exp \left[-k_{k} C_{w}\left(t+\frac{1}{k_{e}} \exp \left(-k_{e} t\right)-\frac{1}{k_{e}}\right)+\left(k_{k} z-h_{b}\right) t\right] & \text { si } C_{i}^{*}(t) \geq z
\end{array}\right.
$$

siendo $h_{b}$ la tasa de riesgo basal, $k_{e}$ la constante de eliminación, $k_{k}$ la tasa de muerte, $C_{w}$ la concentración de xenobiótico en el medio, $C_{i}^{*}(t)$ la concentración interior y z el umbral de tolerancia, común para toda la población y dependiente sólo del compuesto utilizado, por consiguiente uno de los parámetros a estimar. 


\section{Objetivos}

El objetivo de este estudio es realizar un análisis de supervivencia de C. riparius en condiciones de toxicidad aguda frente a triclosán, utilizando para ello una nueva herramienta basada en la teoría GUTS, y explorando la información y posibilidades que aporta esta nueva técnica.

\section{Material y métodos}

\section{Ensayos de supervivencia}

Los ensayos de supervivencia se realizaron con larvas de C. riparius de cuarto estadio criadas en laboratorio. La población tiene su origen en larvas silvestres recolectadas en Valencia (España), cultivadas posteriormente en el laboratorio de la Facultad de Ciencias de la Universidad Nacional de Educación a Distancia (UNED) siguiendo los protocolos estandarizados para los test de toxicidad (USEPA, 2000; OCDE, 2004). El medio de cultivo utilizado estaba compuesto por $\mathrm{CaCl}_{2} 0.5 \mathrm{mM}, \mathrm{NaCl} 1 \mathrm{mM}$, $\mathrm{MgSO}_{4} 1 \mathrm{mM}, \mathrm{NaHCO}_{3} 0.1 \mathrm{mM}, \mathrm{KH}_{2} \mathrm{PO}_{4} 0.025 \mathrm{mM}$ y FeCl $30.01 \mathrm{mM}$ suplementado con comida comercial para peces (TetraMin, Tetra) y hojas de ortiga trituradas. Los cultivos se mantuvieron a $18^{\circ}-20^{\circ} \mathrm{C}$, con ciclos de luz/oscuridad de $16 / 8$ horas y aireación constante.

Se estudió la supervivencia de las larvas frente a triclosán (TCS) (Sigma) utilizando etanol como disolvente. Las concentraciones de TCS a las que se les sometió fueron de 1-1.5-1.75-2-2.5-3 mg/L, más el control con etanol. Por cada una de estas concentraciones se realizaron tres tratamientos (réplicas) independientes; cada uno de ellos constaba de 50 larvas en un recipiente de cristal cubierto de papel de aluminio para evitar la fotodegradación, con orificios para una aireación adecuada. Se contó el número de larvas vivas a las: 0-6-12-18-24-36-42-48-60-66-72-84-90 horas. Las larvas fueron consideradas muertas cuando no reaccionaron al contacto suave con las pinzas. Los individuos muertos fueron retirados.

\section{Análisis de supervivencia}

Los datos obtenidos en los ensayos se utilizaron para ajustar los modelos de la teoría GUTS: el modelo SD y cinco modelos IT correspondientes a cinco funciones de densidad: exponencial (3 parámetros), log-logística, normal, gamma y de Weibull (cuatro parámetros). El ajuste se realizó mediante el método de máxima verosimilitud, a través de la función de log-verosimilitud (Jager et al, 2011):

$$
\ln (\boldsymbol{\theta} \mid y)=\sum_{i=1}^{n+1}\left(y_{i-1}-y_{i}\right) \ln \left(S_{i-1}(\boldsymbol{\theta})-S_{i}(\boldsymbol{\theta})\right)
$$


Siendo $\theta$ el vector de parámetros a estimar, $\mathrm{y}_{i}$ las larvas supervivientes en la medida número $i$, Si $(\theta)$ el valor de la función de supervivencia (en función de los parámetros) para el tiempo y la concentración de la medida $i$, y $n$ el número de medidas realizadas. El valor $n+1$ se refiere a una medida en tiempo infinito en la que no habría supervivientes (Ashauer et al, 2015). Esto permite introducir el término de censura (supervivientes en la última medida realizada).

Los intervalos de confianza se obtuvieron mediante la razón de verosimilitud (Meeker y Escobar, 1995), utilizando un nivel de significación de $\alpha=0.05$.

La elección del modelo que mejor ajusta a los datos se realizó siguiendo el criterio de Akaike. Para facilitar la comparación entre modelos se hallaron también los pesos de Akaike, que dan una estimación de la probabilidad de cada modelo de ser el que mejor ajuste a los datos (Akaike, 1974; Burnham y Anderson, 2002; Symonds y Moussalli, 2011).

Todos los cálculos matemáticos de los modelos GUTS fueron realizados con la ayuda del software MATLAB (The MathWorks, USA).

A modo de comparativa, se calcularon las concentraciones letales a 24 y 48 h con el modelo probit habitual, mediante el software estadístico IBM SPSS.

\section{Resultados}

El mejor ajuste se obtuvo con los modelos IT de cuatro parámetros, en concreto el modelo IT con función de distribución de la tolerancia tipo Weibull fue el mejor según el criterio de Akaike (Tabla 1).

\begin{tabular}{|l|c|c|c|c|c|c|}
\hline Modelo & IT Weibull & IT Normal & IT Gamma & IT log-logística & SD & IT exponencial \\
\hline Peso de Akaike & 0.8176 & 0.1824 & $4.5 \cdot 10^{-8}$ & $5.1 \cdot 10^{-12}$ & $2.9 \cdot 10^{-34}$ & $1.4 \cdot 10^{-80}$ \\
\hline
\end{tabular}

Tabla 1.- Pesos de Akaike de los seis modelos ajustados.

Table 1.- Akaike weights for the six tested models.

Los valores de los parámetros estimados para este modelo de Weibull se recogen en la Tabla 2, siendo $h_{b}$ la tasa de riesgo basal (del control), $k_{e}$ la constante de eliminación, y $\alpha$ y $\beta$ parámetros propios de la función densidad de probabilidad de Weibull.

\begin{tabular}{|l|c|c|c|c|}
\hline TCS & $h_{b}\left(h^{-1}\right)$ & $k_{e}\left(h^{-1}\right)$ & $\alpha(\mathrm{mg} / \mathrm{L})$ & $(-)$ \\
\hline IT Weibull & 0.0031 & 0.0245 & 2.70 & 1.98 \\
\hline I.C. 95\% & $(0.0025,0.0037)$ & $(0.0230,0.0261)$ & $(2.47,2.94)$ & $(1.91,2.05)$ \\
\hline
\end{tabular}

Tabla 2.- Resultados del ajuste de los datos de supervivencia al TCS a un modelo IT de Weibull, e intervalos de confianza (IC) al 95\%.

Table 2.- Results of fitting the survival data for TCS to a Weibull IT model, and $95 \%$ confidence intervals. 
Una vez establecido el mejor modelo según este criterio se pudieron calcular las concentraciones letales de TCS a cualquier tiempo. En la tabla 3 se recogen a modo de ejemplo las LCx a 24 y $48 \mathrm{~h}$.

\begin{tabular}{|l|c|c|c|c|}
\hline IT Weibull & \multicolumn{2}{|c|}{ 24h } & \multicolumn{2}{c|}{ 48h } \\
\hline LCx & LCx (mg/L) & IC 95\% & LCx (mg/L) & IC 95\% \\
\hline LC10 & 1.95 & $(1.64,2.31)$ & 1.25 & $(1.07,1.47)$ \\
LC25 & 3.24 & $(2.77,3.78)$ & 2.08 & $(1.81,2.40)$ \\
LC50 & 5.05 & $(4.81,5.80)$ & 3.24 & $(2.86,3.68)$ \\
LC75 & 7.16 & $(6.30,8.13)$ & 4.60 & $(4.11,5.16)$ \\
LC95 & 10.57 & $(9.43,11.83)$ & 6.80 & $(6.15,7.51)$ \\
\hline
\end{tabular}

Tabla 3.- Concentraciones letales de TCS a 24 y 48 h, según el modelo IT Weibull.

Table 3.- Lethal concentrations of TCS at 24 and $48 \mathrm{~h}$, for the Weibull IT model.

Así mismo, se calculó la tolerancia media de la población al TCS a partir de los parámetros $\alpha=2.70$ y $\beta=1.98 \mathrm{mg} / \mathrm{L}$, obteniendo en este caso un valor de $1.76 \mathrm{mg} / \mathrm{L}$, que representa la concentración escalada interna media de la población, valor a partir del cual la larva muere.

Con los datos de supervivencia a 24 y 48 h se hallaron también las LCx mediante el método probit, recogidas en la tabla 4.

\begin{tabular}{|l|c|c|c|c|}
\hline \multirow{2}{*}{$\begin{array}{l}\text { Probit } \\
\text { LCx }\end{array}$} & \multicolumn{2}{|c|}{ 24h } & \multicolumn{2}{c|}{ 48h } \\
\cline { 2 - 5 } & LCx (mg/L) & IC 95\% & LCx (mg/L) & IC 95\% \\
\hline LC10 & 1.43 & $(0,2.23)$ & 1.189 & $(0.20,1.66)$ \\
LC25 & 2.48 & $(0.13,3.11)$ & 1.843 & $(1.24,2.17)$ \\
LC50 & 3.65 & $(3.03,7.66)$ & 2.571 & $(2.27,2.87)$ \\
LC75 & 4.82 & $(3.83,14.32)$ & 3.298 & $(2.98,3.87)$ \\
LC95 & 6.50 & $(4.794,24.06)$ & 4.344 & $(3.80,5.55)$ \\
\hline
\end{tabular}

Tabla 4.- Concentraciones letales de TCS a 24 y 48 h, según el modelo probit.

Table 4.- Lethal concentrations of TCS at 24 and $48 \mathrm{~h}$, for the probit model.

\section{Discusión}

Se realizó un análisis de la supervivencia de larvas de cuarto estadio de C. riparius frente a TCS. Este tipo de análisis con frecuencia se realiza a través de modelos muy sencillos como el probit, de fácil acceso en la mayoría de los paquetes informáticos de estadística, cuyo objetivo principal suele ser el cálculo de las LCx. Sin embargo, en esta investigación se optó por utilizar modelos matemáticos basados en la teoría GUTS, que facilitan más información sobre el proceso que el mero cálculo de las LCX. 
Se probaron cinco modelos diferentes de la variante IT de esta teoría, así como un modelo SD. De ellos, el modelo IT de Weibull fue con claridad el que mejor ajustaba a los datos, según el criterio de Akaike. Esto es, el modelo matemático que mejor ajusta a los datos de supervivencia supone que la tolerancia umbral al TCS no es la misma en todos los individuos, sino que se distribuye en la población según una distribución de Weibull.

Una vez establecido el modelo pueden obtenerse datos de sumo interés. Se relaciona tanto la concentración externa como el tiempo y la supervivencia (Fig. 3), lo que permite una mayor flexibilidad en los cálculos. Por un lado, las concentraciones y tiempos letales no se ven limitados a los datos utilizados en los experimentos, como puede ocurrir en otros modelos como el probit, sino que es posible obtener las LCx a cualquier tiempo, aunque en ese intervalo temporal en concreto no se hayan recogido datos de supervivencia. Lo mismo podría hacerse fijando la concentración externa y hallando los tiempos letales (LTX) de elección. La flexibilidad de este modelo permite, por ejemplo, obtener información relativa a la supervivencia a exposición a bajas concentraciones y tiempos más largos, aunque los datos no hayan sido recogidos en esas condiciones.

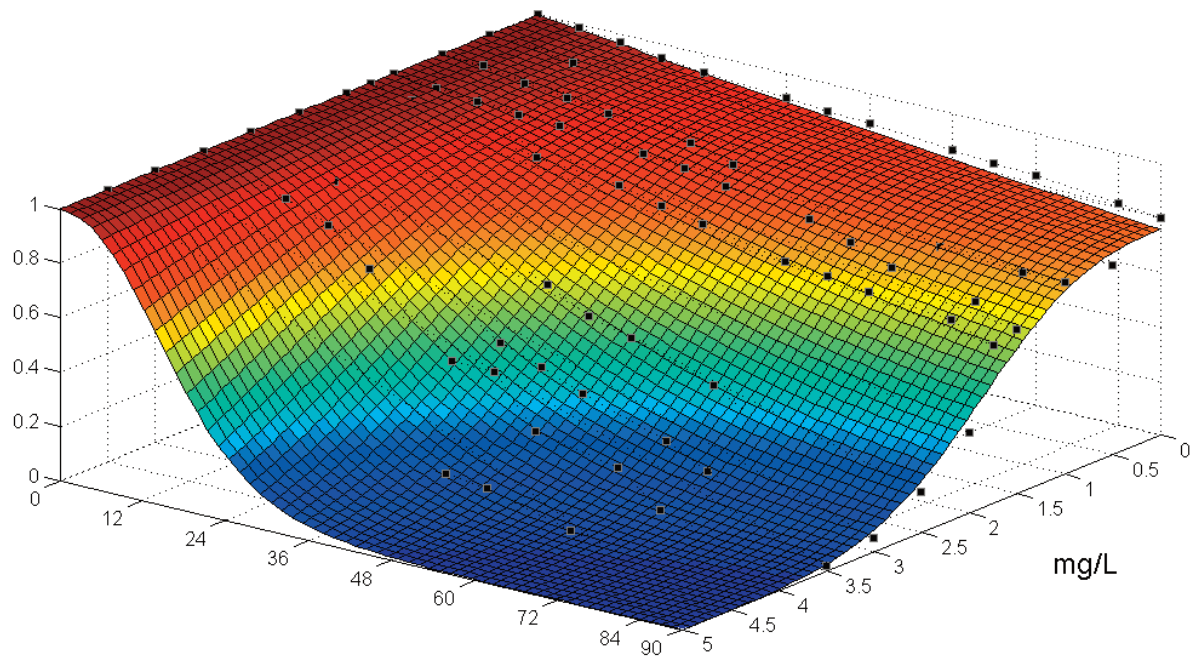

$\mathrm{t}(\mathrm{h})$

Fig. 3.- Superficie de supervivencia en función del tiempo de exposición (horas) y la concentración de TCS (mg/L). Los puntos representan los datos experimentales.

Fig. 3.- Survival surface, as a function of time (hours) and TCS concentration (mg/L). Black dots are experimental data. 
Estos cálculos sencillos de LCx y LTx son sumamente prácticos, pero la información que puede obtenerse del modelo va mucho más allá. Permite el estudio de la distribución de la concentración interna escalada de la población. Se obtuvo un valor de $1,76 \mathrm{mg} / \mathrm{L}$ de TCS para la media poblacional de la concentración escalada interna umbral, valor a partir del cual la larva muere. Tal y como se mencionó en la introducción la concentración escalada interna no es exactamente la concentración interna de TCS, sino que está relacionada con la concentración interna real por el factor de bioconcentración. Para hallar la concentración interna con exactitud y relacionarla con la supervivencia mediante la teoría GUTS se requieren ensayos más complejos, como se ha efectuado ya en algunos estudios utilizando trazadores radiactivos (Nyman et al., 2012).

A pesar de ello, esta media poblacional permite establecer múltiples relaciones de sumo interés: entre la tolerancia a diferentes compuestos, entre pautas de exposición a la misma concentración (continua, por pulsos, etc.), o entre diferentes poblaciones de $C$. riparius de laboratorio y de campo, cultivos de diferentes laboratorios, e incluso permitiría analizar las diferencias estacionales de una misma población. En definitiva, se cuantifica detalladamente la respuesta de la población al compuesto y las diferencias entre individuos, con todo lo que esto conlleva.

Las LCx obtenidas mediante el método probit son similares, aunque ligeramente inferiores, encontrando la mayor diferencia en el LC95 a 24 h: 10.57 mg/L con la teoría GUTS y 6.50 mg/L con el método probit. No se han encontrado datos en la bibliografía que puedan validar uno u otro resultado, no obstante, las características del modelo GUTS, que utiliza todos los datos temporales para hacer la estimación, hacen suponer que sus estimaciones sean más precisas.

Otra de las puertas que abre la teoría GUTS es la de la simulación. Una vez establecido el modelo pertinente (en este caso el modelo IT con distribución de Weibull) pueden simularse nuevos escenarios. Una de las aplicaciones inmediatas es el diseño de experimentos. De esta forma, si en estudios de otra índole (por ejemplo, de genotoxicidad), son necesarias unas condiciones de supervivencia concretas, no es necesario un experimento previo para establecer las concentraciones adecuadas, sino que pueden simularse infinidad de posibilidades en el ordenador (concentraciones constantes, por pulsos, etc.) y elegir el régimen más conveniente. Las posibilidades de combinación de concentraciones y tiempos son ilimitadas. En la Fig. 4 se puede observar una simulación ejemplo que compara la administración de dos pulsos de TCS de $2 \mathrm{mg} / \mathrm{L}$ durante $24 \mathrm{~h}$ con la aplicación de un pulso de $1 \mathrm{mg} / \mathrm{L}$ seguido de otro de $3 \mathrm{mg} / \mathrm{L}$. En ambos casos puede apreciarse en los gráficos la evolución de la concentración interna y la supervivencia. Además de estos gráficos sencillos cada simulación proporciona así mismo una matriz con todos los datos de concentración interna y supervivencia a lo largo del tiempo, con los intervalos de confianza elegidos. 

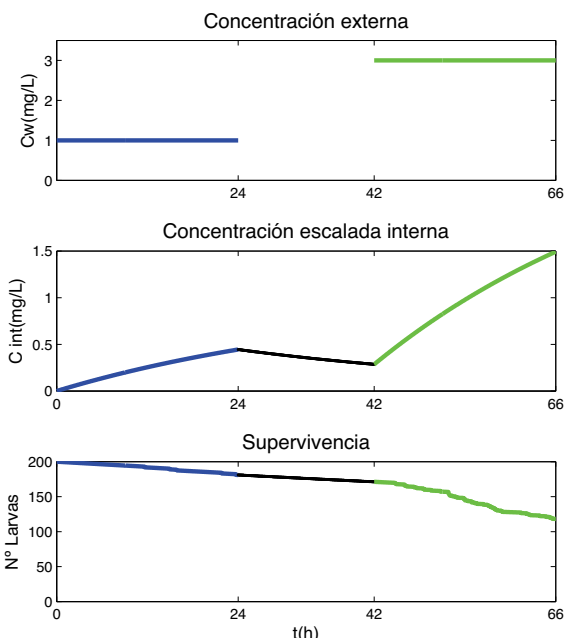
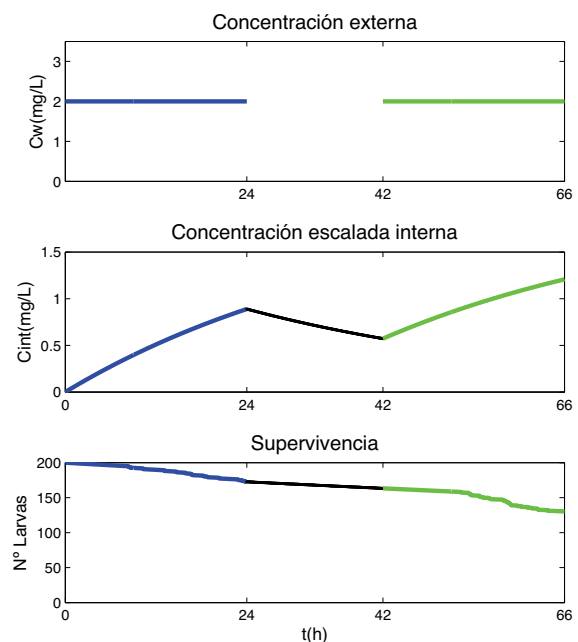

Fig. 4.- Ejemplo de simulación: exposición a dos pulsos de TCS durante 24 h, separados por $18 \mathrm{~h}$ de recuperación. Izqda.: pulsos de $1 \mathrm{mg} / \mathrm{L}$ y $3 \mathrm{mg} / \mathrm{L}$. Dcha.: dos pulsos iguales de $2 \mathrm{mg} / \mathrm{L}$. Pueden apreciarse las diferencias y la evolución en el tiempo tanto de la concentración escalada interna como de la supervivencia.

Fig. 4.- Simulation example: two 24 hour exposure to TCS pulses, separated by a recovery period of $18 \mathrm{~h}$. Left: different pulses of $1 \mathrm{mg} / \mathrm{L}$ and $3 \mathrm{mg} / \mathrm{L}$. Right: two pulses of $2 \mathrm{mg} / \mathrm{L}$. The evolution over time of the scaled internal concentration and the survival function can be seen in the graphs.

Aunque es necesaria más investigación al respecto, otra de las posibilidades que se abre con el modelado es la simulación de escenarios reales. No obstante, son muchas las dificultades que quedan por superar: en este trabajo se utilizan larvas de cuarto estadio que en campo viven en sedimento. Además su densidad se ve afectada por otros factores como, por ejemplo, la lluvia y la supervivencia en cada uno de los estadios influye en la de los demás. Un escenario real es un sistema sumamente complejo y aunque ya se han dado algunos pasos hacia la simulación (Ashauer et al., 2012; Urien, 2017) la multitud de factores que intervienen en campo hacen que sea necesaria la participación de expertos en distintas áreas (biólogos, ecólogos, ingenieros expertos en hidrología, matemáticos, etc.)."

\section{Conclusiones}

Los modelos matemáticos y la simulación soy hoy en día una herramienta de análisis de gran valor, que se está introduciendo rápida y sólidamente en la mayoría de las disciplinas científico-técnicas, económicas, sociales, etc. Aunque su adaptación a la 
ecotoxicología se está produciendo más lentamente ya han aparecido diversos modelos, como los basados en la teoría GUTS, que permiten obtener gran cantidad de información de los ensayos ecotoxicológicos habituales, y extrapolar dicha información a otros escenarios. En el presente estudio se ha analizado la aplicación de esta teoría a la supervivencia de C. riparius frente a TCS, obteniendo datos relevantes y una cuantificación precisa del efecto de la sustancia, lo que abre así mismo la puerta a nuevas investigaciones: comparación de diversas poblaciones de C. riparius, simulación de nuevos escenarios, inclusión en los estudios futuros de las condiciones de escenario real (campo), etc.

Es imprescindible la colaboración de investigadores de diferentes disciplinas (biólogos, ecólogos, matemáticos, informáticos, físicos, etc.), aportando cada uno de ellos su visión en un ambiente multidisciplinar y sumamente enriquecedor.

\section{Bibliografía}

Akaike, H. 1974. A new look at the statistical model identification. IEEE Transactions on Automatic Control. 19(6): 716-723.

Ashauer, R., Thorbek, P., Warinton, J.S., Wheeler, J.R., Maund, S. 2013. A method to predict and understand fish survival under dynamic chemical stress using standard ecotoxicity data. Environmental Toxicology and Chemistry / SETAC. 32(4): 954-965.

Berg, M.B., Hellenthal, R.A. 1992. The role of Chironomidae in energy flow of a lotic ecosystem. Aquatic Ecol. 26(2-4): 471-476.

Bhattacharyay, G., Sadhu, A.K., Mazumdar, A., Chaudhuri, P.K. 2005. Antennal deformities of Chironomid larvae and their use in biomonitoring of heavy metal pollutants in the river Damodar of West Bengal, India. Environ. Monit. Assess. 108: 67-84.

Bimalendu, B. Nath. 2018. Extracellular hemoglobin and environmental stress tolerance in Chironomus larvae. J. Limnol. 77(1): 104-112.

Burnham, K.P, Anderson, D.R. 2002. Model selection and multimodel inference: A practical information-theoretic approach. Springer. New York.

Chen, J., Ahn, K.C., Gee, N.A., Gee, S.J., Hammock, B.D., Lasley, B.L. 2007. Antiandrogenic properties of parabens and other phenolic containing small molecules in personal care products. Toxicol. Appl. Pharmacol. 221(3): 278-284.

Dann, A.B., Hontela, A. 2011. Triclosan: environmental exposure, toxicity and mechanisms of action. J. Appl. Toxicol. 31: 285-311.

Gee, R.H., Charles, A., Taylor, N., Darbre, P.D. 2008. Oestrogenic and androgenic activity of triclosan in breast cancer cells. J. Appl. Toxicol. 28: 78-91. 
Jager, T., Albert, C., Preuss, T.G., Ashauer, R. 2011. General Unified Threshold Model of Survival - a Toxicokinetic-Toxicodynamic Framework for Ecotoxicology. Environ. Sci. Technol. 45(7): 2529-2540.

Helbing, C.C., Van Aggelen, G., Veldhoen, N. 2010. Triclosan affects thyroid hormone-dependent metamorphosis in anurans. Toxicol. Sci. 119(2): 417-418.

Martínez-Paz, P., Morales, M., Urien, J., Morcillo, G., Martínez-Guitarte, J.L. 2017. Endocrinerelated genes are altered by antibacterial agent triclosan in Chironomus riparius aquatic larvae. Ecotoxicol. Environ. Saf. 140: 185-190.

Meeker, W.Q, Escobar, L.A. 1995. Teaching about Approximate Confidence Regions Based on Maximum Likelihood Estimation. Am. Stat. 49: 48-53.

Morales, M., Planelló, R., Martínez-Paz, P., Herrero, O., Cortés, E., Martínez-Guitarte, J.L., Morcillo, G. 2011. Characterization of Hsp70 gene in Chironomus riparius: expression in response to endocrine disrupting pollutants as a marker of ecotoxicological stress. Comparative Biochemistry and Physiology. Part C: Toxicol. Pharmacol. Endocrinol. 153(1): 150-158.

Moller Pillot, H.K.M. 2009. Chironimidae larvae II: Biology and Ecology of the Chironomini.KNNV. Amsterdam.

Nyman, A.M., Schirmer, K., Ashauer, R. 2012. Toxicokinetic-toxicodynamic modelling of survival of Gammarus pulex in multiple pulse exposures to propiconazole: model assumptions, calibration data requirements and predictive power. Ecotoxicol. 21(7): 1828-1840.

OECD 2004, Test No. 218: Sediment-Water Chironomid Toxicity Using Spiked Sediment, OECD Guidelines for the Testing of Chemicals, Section 2, OECD Publishing, Paris.

OECD 2004, Test No. 219: Sediment-Water Chironomid Toxicity Using Spiked Water, OECD Guidelines for the Testing of Chemicals, Section 2, OECD Publishing, Paris.

OECD 2010, Test No. 233: Sediment-Water Chironomid Life-Cycle Toxicity Test Using Spiked Water or Spiked Sediment, OECD Guidelines for the Testing of Chemicals, Section 2, OECD Publishing, Paris.

OECD 2011, Test No. 235: Chironomus sp., Acute Immobilisation Test, OECD Guidelines for the Testing of Chemicals, Section 2, OECD Publishing, Paris,

Oliveira, R., Domingues, I., Grisolia, C.K., Soares, A. 2009. Effects of triclosan on zebrafish early life stages and adults. Environ. Sci. Pollut. Res. 16(6): 679-688.

Park, S., Choi, J. 2009. Genotoxic effects of nonylphenol and bisphenol. A exposure in aquatic biomonitoring species: freshwater crustacean, Daphnia magna, and aquatic midge, Chironomus riparius. Bull. Environ. Contam. Toxicol. 83: 463-468.

Planelló, R., Servia, M.J., Gómez-Sande, P., Herrero, Ó., Cob,o F., Morcillo, G. 2015. Transcriptional responses, metabolic activity and mouthpart deformities in natural populations of Chironomus riparius larvae exposed to environmental pollutants. Environ. Toxicol. 30(4): 383-395. 
Rodricks, J.V., Swenberg, J.A., Borzelleca, J.F., Maronpot, R.R., Shipp, A.M. 2010. Triclosan: a critical review of the experimental data and development of margins of safety for consumer products. Critical Rev. Toxicol. 40(5): 422-484.

Sandborgh-Englund, G., Adolfsson-Erici, M., Odham, G., Ekstrand, J. 2006. Pharmacokinetics of triclosan following oral ingestion in humans. J. Toxicol. Environ. Health 69(Part A.): 1861 1873

Symonds, M., Moussalli, A. 2011. A brief guide to model selection, multimodel inference and model averaging in behavioural ecology using Akaike's information criterion. Behav. Ecol. Sociobiol. 65(1): 13-21.

Toms, L.L., Allmyr, M., Mueller, J.F., Adolfsson-Erici, M., McLachlan, M., Murby, J., Harden, F.A. 2011. Triclosan in individual human milk samples from Australia. Chemosphere. 85: 16821686.

Urien, J. 2017. Análisis de supervivencia de Chironomus riparius expuesto a sustancias que contaminan el medio ambiente. Tesis doctoral. UNED. Madrid.

Xu, X., Lu, Y., Zhang, D., Wang, Y., Zhou, X., Xu, H., Mei, Y. 2015. Toxic Assessment of Triclosan and Triclocarban on Artemia salina. Bull. Environ. Contam. Toxicol. 95: 728-733.

Wiseman, S.B., Anderson, J.C., Liber, K., Giesy, J.P. 2013. Endocrine disruption and oxidative stress in larvae of Chironomus dilutus following short-term exposure to fresh or aged oil sands process-affected water. Aquat. Toxicol. 142: 414-421.

Fecha de recepción/ Date of reception: 12/02/2019 Fecha de aceptación / Date of acceptance: 10/05/2019 